GA-A23847

\title{
MULTI-MODE ERROR FIELD CORRECTION ON THE DIII-D TOKAMAK
}

\author{
by \\ J.T. SCOVILLE and R.J. LA HAYE
}

OCTOBER 2002 


\section{DISCLAIMER}

This report was prepared as an account of work sponsored by an agency of the United States Government. Neither the United States Government nor any agency thereof, nor any of their employees, makes any warranty, express or implied, or assumes any legal liability or responsibility for the accuracy, completeness, or usefulness of any information, apparatus, product, or process disclosed, or represents that its use would not infringe privately owned rights. Reference herein to any specific commercial product, process, or service by trade name, trademark, manufacturer, or otherwise, does not necessarily constitute or imply its endorsement, recommendation, or favoring by the United States Government or any agency thereof. The views and opinions of authors expressed herein do not necessarily state or reflect those of the United States Government or any agency thereof. 


\title{
MULTI-MODE ERROR FIELD CORRECTION ON THE DIII-D TOKAMAK
}

\author{
by \\ J.T. SCOVILLE and R.J. LA HAYE
}

This is a preprint of a paper to be submitted for publication in Nucl. Fusion.

\author{
Work supported by \\ the U.S. Department of Energy under \\ Contract No. DE-AC03-99ER54463
}

GENERAL ATOMICS PROJECT 30033 OCTOBER 2002 


\begin{abstract}
Error field optimization on DIII-D tokamak [Luxon, J.L., Nucl. Fusion 42 (2002) 814] plasma discharges has routinely been done for the last ten years with the use of the external " $n=1$ coil" or the "C-coil". The optimum level of correction coil current is determined by the ability to avoid the locked mode instability and access previously unstable parameter space at low densities. The locked mode typically has toroidal and poloidal mode numbers $n=1$ and $m=2$, respectively, and it is this component that initially determined the correction coil current and phase. Realization of the importance of nearby $n=1$ mode components $m=1$ and $m=3$ has led to a revision of the error field correction algorithm. Viscous and toroidal mode coupling effects suggested the need for additional terms in the expression for the radial "penetration" field $B_{\text {pen }}$ that can induce a locked mode. To incorporate these effects, the low density locked mode threshold database was expanded. A database of discharges at various toroidal fields, plasma currents, and safety factors was supplemented with data from an experiment in which the fields of the $n=1$ coil and C-coil were combined, allowing the poloidal mode spectrum of the error field to be varied. A multivariate regression analysis of this new low density locked mode database was done to determine the low density locked mode threshold scaling relationship $n_{\mathrm{e}} \propto B_{\mathrm{T}}^{-0.01} q_{95}^{-0.79} B_{\text {pen }}$ and the coefficients of the poloidal mode components in the expression for $B_{\text {pen }}$. Improved plasma performance is achieved by optimizing $B_{\text {pen }}$ by varying the applied correction coil currents.
\end{abstract}




\section{INTRODUCTION}

\section{A. Background}

Small errors in the symmetry of the magnetic field of a tokamak (error fields) can drive instabilities such as locked modes. The locked mode is a non-rotating helical magnetic field perturbation that leads to loss of energy confinement and plasma disruption. The typical low density locked mode grows in a fixed toroidal location as the density is reduced, without any previously observed rotating modes in the plasma. Locked modes can also result when rotating modes are slowed by their interaction with resistive vessel walls or an external static error field.

The investigation of error fields and their effect on plasma confinement has been underway since the 1970s, when external coils were used to apply resonant magnetic perturbations (RMPs) in the PULSATOR [1] and ATC [2] tokamaks. More recent experiments in the early 1990s on the COMPASS-C [3,4], DIII-D [5,6] and JET [7] tokamaks have investigated the locked mode phenomenon in detail, measuring mode structure and the dependence on various plasma parameters. In particular, modes that grow at low $\beta$ and low density without a rotating precursor have received considerable attention from experimentalists [3-7] as well as theorists [8-12].

The interaction of a rotating plasma with an error field is a complex trade-off between the driving forces (e.g., from neutral beams), the viscous forces, and the electromagnetic torques from the resistive wall and error field that try to reduce the rotation speed [9]. From theory, reducing the error field by applying a correcting field of the proper amplitude and phase is expected to expand the stable operating parameter space of the tokamak [9-11,13]. A momentum source such as neutral beam injection is also important, since higher rotation speed is a stabilizing effect if $\beta$ is not too high [14]. Rotating magnetic islands (e.g., tearing modes) "slipping" through the plasma experience a velocity shear that tends to reduce the size of the island $[10,12]$. However, a sufficiently large static error field can overcome the rotational stabilization, slow and lock the islands, and drive them to much larger amplitudes, with severe detrimental effects on the plasma.

\section{B. History of Error Field Correction on DIII-D}

To optimize the error field of a tokamak, the mode structure, amplitude, and phase of hardware-related field contributions should be known. Even without this knowledge, initial experiments on DIII-D had some success using the " $n=1$ coil", a large perturbation coil mounted on top of the machine and offset radially to be directly above the center of the 
plasma current, as shown in Fig. 1. A scan of the low density locked mode threshold was performed and clearly showed that at an optimum $n=1$ coil current, an improvement in the minimum accessible density was obtained [5]. The ability to avoid the locked mode was limited, however, since the $n=1$ coil poloidal mode spectrum and phase could not be changed.

Direct measurement of the $n=1$ and $n=2$ error fields produced by the fieldshaping coils on DIII-D [15] led to the design of the C-coil to enable significant modification of the reported error fields. The C-coil (Fig. 1) consists of six midplane saddle coils that are typically connected in three diametrically opposite pairs to generate odd toroidal mode numbers (primarily $n=1$ ) for error field correction. The first C-coil experiments attempted to affect the $m, n=2,1 \mathrm{com}$ ponent of the error field, since this is the mode most often observed. The mode exists on the resonant surface defined by

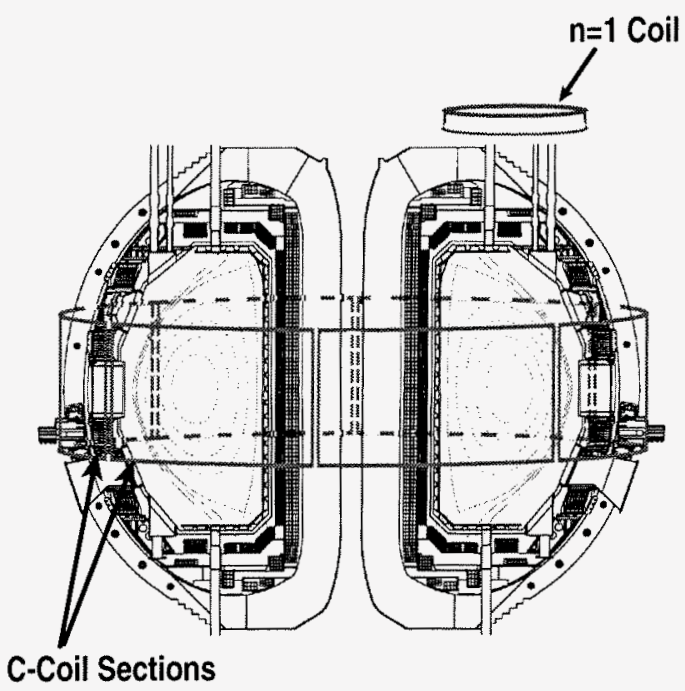

Fig. 1. Cross section of the DIII-D tokamak showing the $n=1$ coil and the C-coil error field correction coils. $q=m / n=2$, where $q$ is the safety factor. Altering the 2,1 component by optimizing both the phase and amplitude of the C-coil indeed resulted in an expanded stable operating parameter space [16]. Simply optimizing the 2,1 component at the $q=2$ surface was not enough, however, as it became evident that mode-coupling from components at the $q=1$ and $q=3$ surfaces could increase the 2,1 component [4,10]. Simultaneous reduction of the $m=1, m=2$, and $m=3$ components of the $n=1$ mode should be the goal of an efficient error field correction system and recent studies and analyses for the ITER design have adopted this position $[17,18]$.

Section 2 of this paper discusses the theoretical motivation for a multi-mode error field correction system and develops an expression for the radial penetration field $B_{\text {pen }}$ that must be optimized. Section 3 presents experimental results with an analysis that determines the mode-coupling coefficients contributing to $B_{\text {pen }}$ and the scaling law that predicts the low density locked mode threshold. Section 4 summarizes the multi-mode error field correction efforts on DIII-D and points out the need to better correct error fields in high beta plasmas near the resistive wall limit. 


\section{THEORY OF LOCKED MODES}

\section{A. Torque Balance and Scaling Laws}

Locked modes in tearing stable plasmas are caused by torques from helical currents at resonant flux surfaces. The helical current sheets are a resonant response of the plasma to an external field perturbation. Islands can grow at the resonant surface if the external perturbation exceeds a critical value. Below this threshold, magnetic reconnection is rotationally suppressed as the small islands "slip" through, or are partially dragged along with the rotating plasma and do not grow. A locked mode results from a change of state from slipping to locked, and is induced by error field "penetration" $[9,10,12]$.

A rational flux surface defined by $q=m / n$ acts as a thin conducting layer. A helical current is induced on that layer in response to a component of the radial field $B_{\mathrm{rmn}}$. As shown in Fig. 2, a fraction of the resistively phase-shifted eddy current, $\delta J_{\theta}$, interacts with the error field to produce a $\delta J_{\theta} \times B_{\mathrm{r}}$ torque that can be written as $[10,12]$

$$
T_{\phi \mathrm{mn}}=-R\left(\frac{B_{\mathrm{rmn}}^{2}}{\mu_{0}}\right)\left(\frac{r_{\mathrm{mn}}}{\mathrm{qR}}\right)\left(2 \pi R 2 \pi r_{\mathrm{mn}}\right)\left(\frac{1}{\omega \tau_{\mathrm{rec}}}\right),
$$

where $R$ is the major radius, $r_{\mathrm{mn}}$ is the minor radius of the $q=m / n$ surface, $\omega$ is the rotation frequency, $\tau_{\text {rec }}$ is the reconnection time, governed by the plasma resistivity, and the phase shift between the eddy current and the radial field is $\tan ^{-1}\left(1 / \omega \tau_{\text {rec }}\right)$. Thus, for a slipping plasma, the error field torque at $q=2$ is $T_{\phi} \propto B_{\mathrm{r} 21}^{2} / \omega \tau_{\mathrm{rec}}$. In steady state, balancing the driving torque (e.g., from neutral beams) against the error field torque and the viscosity leads to an expression for the frequency at the $q=2$ surface:

$$
\frac{\omega}{\omega_{0}} \approx \frac{1}{2}+\frac{1}{2} \sqrt{1-\frac{C B_{\mathrm{r} 21}^{2} \tau_{\mathrm{v}}}{\omega_{0}^{2} \tau_{\mathrm{A}}^{2} B_{\mathrm{T}}^{2} \tau_{\mathrm{rec}}}},
$$

where $\omega_{0}$ is the natural frequency of rotation if no error field existed, $\tau_{v}$ is the viscous time, $\tau_{\mathrm{A}}$ is the Alfvén time, and $C$ is a constant of proportionality depending only on geometric terms. Error field penetration occurs when the rotation frequency has decreased to half the natural frequency, at which point the penetration field is defined by

$$
\frac{B_{\mathrm{pen}}}{B_{\mathrm{T}}} \propto \omega_{0} \tau_{\mathrm{A}}\left(\frac{\tau_{\mathrm{rec}}}{\tau_{\mathrm{v}}}\right)^{1 / 2}
$$




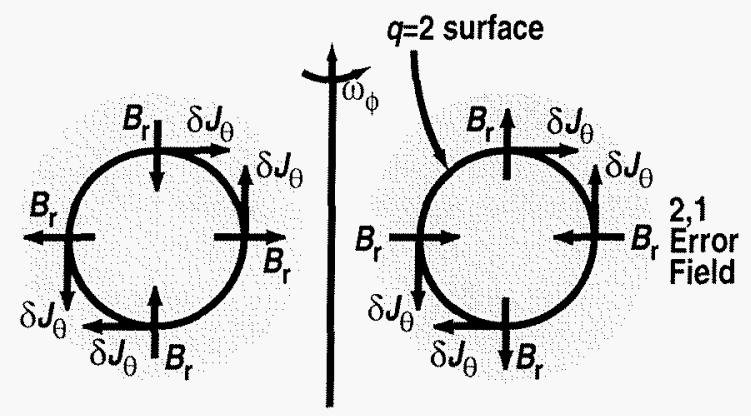

Fig. 2. The $m, n=2,1$ error field component $B_{r}$ produces a helical current sheet $J_{\theta}$ on the $q=2$ surface. The phase-shifted component $\delta J_{\theta}$ interacts with $B_{r}$ to create a torque that reduces the rotation frequency $\omega_{\phi}$.

Following Ref. [6], some assumptions are made to convert Eq. (3) into a relationship between the Ohmic low density locked mode threshold and the error field. The natural frequency is assumed to be the electron drift frequency, $\omega_{0} \approx \omega_{\mathrm{De}} \propto T_{\mathrm{e}} / B_{\mathrm{T}} a^{2}$. Spitzer resistivity deftermines the electron temperature in Ohmic plasmas, $T_{\mathrm{e}} \propto\left(I_{\mathrm{p}} / a^{2}\right)^{4 / 5}\left(\tau_{\mathrm{E}} / n_{\mathrm{e}}\right)^{2 / 5}$, and assuming neo-Alcator scaling, the energy confinement is linear with density, $\tau_{\mathrm{E}} \propto n_{\mathrm{e}} a R^{3 / 2}$. The reconnection (tearing) time is determined by resistivity, $\tau_{\text {rec }} \approx \tau_{\mathrm{R}}^{3 / 5} \tau_{\mathrm{A}}^{2 / 5}$, where $\tau_{\mathrm{R}} \propto T_{\mathrm{e}}^{3 / 2} a^{2}$ is the resistive time and $\tau_{\mathrm{A}}=\left(R / B_{\mathrm{T}}\right) \sqrt{\mu_{0} m_{\mathrm{i}} n_{\mathrm{e}}}$ is the Alfvén time. Ion-ion collisions govern the viscosity $\tau_{\mathrm{v}} \approx a^{2} / \rho_{\theta \mathrm{i}}^{2} v_{\mathrm{ii}} \propto B_{\mathrm{T}}^{2} T_{\mathrm{e}}^{1 / 2} / n_{\mathrm{e}}$, where $\rho_{\theta \mathrm{i}}$ is the ion poloidal gyroradius, $v_{\mathrm{ii}}$ is the ion-ion collision frequency, and we have assumed $T_{\mathrm{e}}=T_{\mathrm{i}}$. For a given value of $q$ we have $I_{\mathrm{p}} \propto B_{\mathrm{T}}$, and thus the penetration field from Eq. (3) can be written

$$
\frac{B_{\text {pen }}}{B_{\mathrm{T}}} \propto n_{\mathrm{e}}^{11 / 10} B_{\mathrm{T}}^{-56 / 25}, \quad \text { or approximately } \quad n_{\mathrm{e}} \propto B_{\text {pen }} B_{\mathrm{T}}
$$

It should be pointed out that Eq. (4) does not include plasma current or safety factor, and that the adoption of a different set of assumptions will produce a different scaling.

\section{B. Mode-Coupling}

Viscous coupling and toroidal coupling are two independent mechanisms whereby a mode resonant on one rational flux surface can interact with a neighboring surface.

In viscous coupling, a torque on the $q=1$ or $q=3$ surface, for example, can exert a force on the $q=2$ surface through plasma viscosity. The total torque at $q=2$ is just the sum of the torques, and is not sensitive to the relative mode phases:

$$
T_{\phi} \propto w_{11} B_{\mathrm{r} 11}^{2}+B_{\mathrm{r} 21}^{2}+w_{31} B_{\mathrm{r} 31}^{2}
$$


where $w_{11}$ and $w_{31}$ are normalized weights for the $m, n=1,1$ and 3,1 error field components.

In toroidal coupling, helical currents flowing on the $q=1$ and $q=3$ surfaces to oppose penetration of the $B_{\mathrm{r} 11}$ and $B_{\mathrm{r} 31}$ error field components produce sidebands at $q=2$ from the toroidicity. As a result, an effective $B_{\mathrm{r} 21}$ can exist even if the applied $B_{\mathrm{r} 21}$ is nulled. In this case, the phase difference between modes is important and a vector sum is necessary:

$$
\vec{B}_{\mathrm{r} 21}^{\mathrm{eff}}=u_{11} \bar{B}_{\mathrm{r} 11}+\bar{B}_{\mathrm{r} 21}+u_{31} \bar{B}_{\mathrm{r} 31}
$$

where $u_{11}$ and $u_{31}$ are normalized geometric coupling coefficients.

Combining Eqs (5) and (6) yields the mode-coupled equation for the torque induced by the penetration field $B_{\text {pen }}$ :

$$
\begin{aligned}
T_{\phi} \propto B_{\mathrm{pen}}^{2}= & w_{11} B_{\mathrm{r} 11}^{2}+\left(u_{11} B_{\mathrm{r} 11 \mathrm{~s}}+B_{\mathrm{r} 21 \mathrm{~s}}+u_{31} B_{\mathrm{r} 31 \mathrm{~s}}\right)^{2} \\
& +\left(u_{11} B_{\mathrm{r} 11 \mathrm{c}}+B_{\mathrm{r} 21 \mathrm{c}}+u_{31} B_{\mathrm{r} 31 \mathrm{c}}\right)^{2}+w_{31} B_{\mathrm{r} 31}^{2}
\end{aligned}
$$

where the sine and cosine components of the toroidal angle (denoted by subscripts $\mathrm{s}$ and $\mathrm{c}$ ) of each error field mode have been used to allow for phase differences in the toroidal coupling. It is the $B_{\text {pen }}$ defined in Eq. (7) that must be minimized for optimal modecoupled error field correction [17]. 


\section{EXPERIMENTAL RESULTS}

\section{A. Very Low Density Regime Accessed}

The $n=1$ coil creates a field with a fixed poloidal mode spectrum, as does the C-coil, but by combining the two coils, the spectrum can be changed. Taking advantage of this fact, an experiment was done in which the $n=1$ coil and C-coil currents were varied during plasma discharges to produce a variety of spectra to test multi-mode error field correction. The density was allowed to decay to find the locked mode threshold as a function of the error field correction coil currents. An example is shown in Fig. 3, where the typical signature for a locked mode is observed. The density decays until the soft X-ray (SXR) sawteeth suddenly disappear at $2.1 \mathrm{~s}$ into the discharge. Simultaneously, the locked mode detector (measuring non-axisymmetric radial magnetic fields via saddle loops) shows a sudden increase and the density decays even faster due to the very poor particle confinement caused by the locked mode. The correction coil currents for this discharge (88613) are zero, establishing the "natural" locked mode density threshold from intrinsic error fields.

Another discharge (88619) is shown in Fig. 4, where the $n=1$ coil and C-coils were used together to modify the magnitude of several low order modes simultaneously. With the correct combination of $n=1$ coil and C-coil currents, the locked mode was completely avoided. The very low densities achieved in these discharges, however, allowed the acceleration of non-thermal electrons (runaways). The runaway bursts are seen as periodic spikes on the SXR and neutron scintillator signals (from hard x-rays). Many discharges exhibited similar behavior as the density fell below a value of approximately $n_{\mathrm{e}} \approx 5.0 \times 10^{12} \mathrm{~cm}^{-3}$. The runaway bursts induce plasma current disruptions and effectively establish a new lower boundary to the stable density limit in DIII-D, a boundary determined by super-thermal electrons, not locked modes.

\section{B. Locked Mode Threshold Scaling and the Error Field, $B_{p e n}$}

The low density Ohmic locked mode threshold database contains four sets of data with different values of $I_{\mathrm{p}}, B_{\mathrm{T}}, q_{95}$, and correction coil currents. All the data discussed here corresponds to typical DIII-D plasmas with left-handed helicity (positive $I_{\mathrm{p}}$ and negative $B_{\mathrm{T}}$ ), although similar data was collected for right-handed helicity plasmas. A multivariate regression analysis of the database was done to determine the unknown components of the penetration field $B_{\text {pen }}$ and the scaling law parameters that define the dependence of the low density locked mode threshold on toroidal field, safety factor, and $B_{\text {pen }}$. Several fits of the 


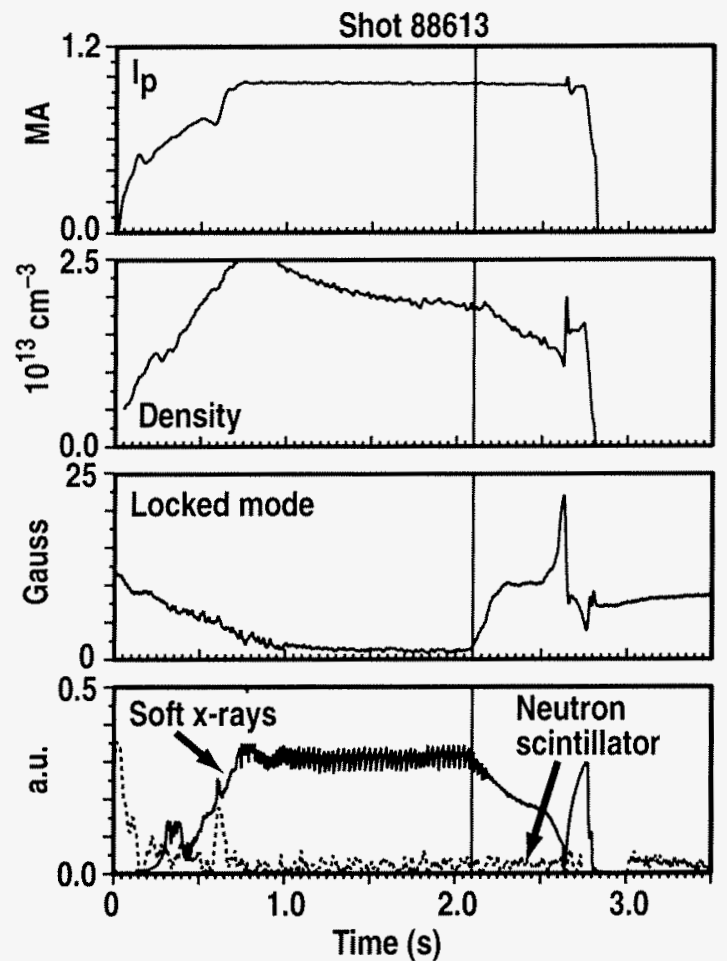

Fig. 3. Plasma current, electron density, radial field from the locked mode detector, soft x-rays, and the neutron scintillator signal from discharge 88613 , with no error field correction. As the density drops below $2.0 \times 10^{13} \mathrm{~cm}^{-3}$, a locked mode grows up at $2.1 \mathrm{~s}$, as evident by the sudden increase in the $n=1$ radial magnetic field measured by saddle loops (locked mode detector) and the disappearance of the soft $x$-ray sawteeth.

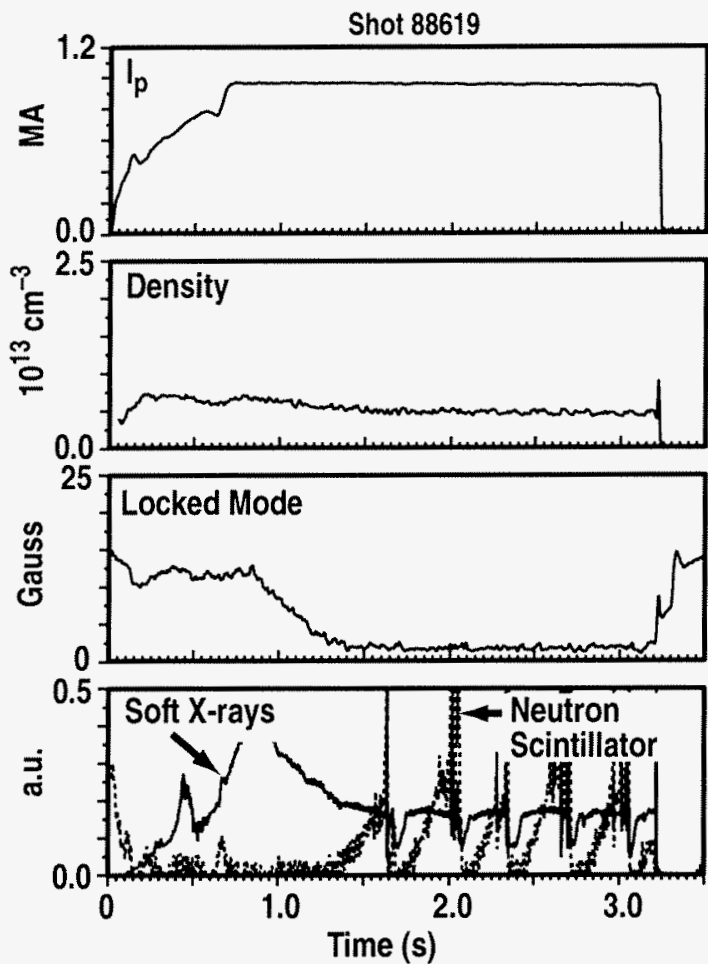

Fig. 4. Discharge 88619 , showing the same signals and plotted on the same scales as in Fig. 3. In this discharge, an optimum mix of correction coil currents from the $n=1$ coil and C-coil allows very low density without a locked mode. The discharge is limited by the onset of runaway electron bursts, evident on the neutron scintillator that responds to high energy $\mathrm{x}$-rays.

form $n_{\mathrm{LM}} \propto B_{\text {pen }}^{\mathrm{x}}$ were tried, varying the value of $\mathrm{x}$, and the best fit to the data consistent with the theory suggested by Eq. (4) was achieved with the form

$$
n_{\mathrm{LM}}=C B_{\mathrm{pen}}
$$

where $C$ depends on the values of $B_{\mathrm{T}}$ and $q_{95}$ (no explicit $I_{\mathrm{p}}$ dependence):

$$
C \propto B_{\mathrm{T}}^{\alpha} q_{95}^{\beta}
$$

The methodology assumed was to first determine the error field contributions from known coil variations and then apply a correcting term using the external coils. Since the toroidal field coil contributions were unknown, an additional term that varies with toroidal field was then included. The relative positions of the poloidal field coils were measured magnetically and this data was used to calculate the low order error field components from these coils [15]. Similarly, the correction coil contributions are also calculated from the 
known geometry of the coils. From Eq. (7), the components $B_{\mathrm{mn}}$ of the error field $B_{\text {pen }}$ are then written as a sum of the known error fields from the poloidal field coils and correction coils, $B_{\mathrm{mn} 0}$, and an unknown term that is assumed to be proportional to toroidal field, $b_{\mathrm{mn}} B_{\mathrm{T}}$ :

$$
\vec{B}_{\mathrm{rmn}}=\bar{B}_{\mathrm{mn} 0}+\vec{b}_{\mathrm{mn}} B_{\mathrm{T}}
$$

Since the sources of the error field components determined by $b_{\mathrm{mn}}$ are presently unknown, they are determined empirically, along with the mode-coupling coefficients in Eq. (7), by regression analysis of subsets of the database with similar values of $q_{95}$. Fitting for the mode-coupling coefficients relies on the fact that the database includes many discharges with different combinations of $\mathrm{C}$-coil and $n=1$ coil currents, thus varying the relative contributions of various mode components significantly. Combining Eqs (7) through (10) shows the complete set of dependent variables that make up the low density locked mode threshold scaling relationship:

$$
\begin{aligned}
n_{\mathrm{LM}}= & C B_{\mathrm{T}}^{\alpha} q_{95}^{\beta}\left\{w_{11}\left(B_{110}+b_{11} B_{T}\right)^{2}+\left[u_{11}\left(B_{110 \mathrm{~s}}+b_{11 \mathrm{~s}} B_{T}\right)+\left(B_{210 \mathrm{~s}}+b_{21 \mathrm{~s}} B_{\mathrm{T}}\right)+u_{31}\left(B_{310 \mathrm{~s}}+b_{31 \mathrm{~s}} B_{\mathrm{T}}\right)\right]^{2}\right. \\
& \left.+\left[u_{11}\left(B_{110 \mathrm{c}}+b_{11 \mathrm{c}} B_{\mathrm{T}}\right)+\left(B_{210 \mathrm{c}}+b_{21 \mathrm{c}} B_{\mathrm{T}}\right)+u_{31}\left(B_{310 \mathrm{c}}+b_{31 \mathrm{c}} B_{\mathrm{T}}\right)\right]^{2}+w_{31}\left(B_{310}+b_{31} B_{T}\right)^{2}\right\}^{1 / 2}
\end{aligned}
$$

where $b_{11}^{2}=b_{11 \mathrm{~s}}^{2}+b_{11 \mathrm{c}}^{2}$. This equation is solved to minimize $\left(n-n_{\mathrm{LM}}\right)^{2}$ using regression analysis on a data set containing a wide range of values to find the 13 quantities $b_{\mathrm{x}}, u_{\mathrm{x}}$, $w_{\mathrm{x}}, C, \alpha$, and $\beta$.

The results of the regression analysis of Eq. (11) are the scaling law for the low density locked mode threshold and the expression for the error field $B_{\text {pen }}$. The scaling law is found to be

$$
n_{\mathrm{LM}}=(0.60 \pm 0.07)\left(\frac{B_{\mathrm{T}}}{2.1 \mathrm{~T}}\right)^{-0.01 \pm 0.27}\left(\frac{q_{95}}{3.3}\right)^{-0.79 \pm 0.55} B_{\mathrm{pen}}
$$

with $n_{\mathrm{LM}}$ in units of $10^{13} \mathrm{~cm}^{-3}$ and $B_{\text {pen }}$ in Gauss. Separate fits for $B_{\text {pen }}$ at high $q$ and low $q$ result in solutions for the two regimes:

$q_{95}=3.2$

$$
B_{\mathrm{pen}}^{2}=0.28 B_{\mathrm{r} 11}^{2}+\left(0.25 B_{\mathrm{r} 1 \mathrm{~s}}+B_{\mathrm{r} 21 \mathrm{~s}}-0.05 B_{\mathrm{r} 31 \mathrm{~s}}\right)^{2}
$$

$$
\begin{aligned}
& +\left(0.25 B_{\mathrm{r} 1 \mathrm{lc}}+B_{\mathrm{r} 21 \mathrm{c}}-0.05 B_{\mathrm{r} 3 \mathrm{lc}}\right)^{2}+0.51 \mathrm{~B}_{\mathrm{r} 31}^{2} \\
q_{95}=4.6 \quad B_{\mathrm{pen}}^{2}=0.32 & B_{\mathrm{r} 11}^{2}+\left(0.21 B_{\mathrm{r} 1 \mathrm{ls}}+B_{\mathrm{r} 21 \mathrm{~s}}+0.30 \mathrm{~B}_{\mathrm{r} 31 \mathrm{~s}}\right)^{2} \\
& +\left(0.21 B_{\mathrm{r} 11 \mathrm{c}}+B_{\mathrm{r} 21 \mathrm{c}}+0.30 B_{\mathrm{r} 31 \mathrm{c}}\right)^{2}+0.41 \mathrm{~B}_{\mathrm{r} 31}^{2}
\end{aligned}
$$


The scaling law of Eq. (12) expresses the low density locked mode threshold in terms of the macroscopic parameters $B_{\mathrm{T}}$ and $q_{95}$. Similar scaling studies have been done in JET error field experiments [19], and an excellent comparison of the locked mode threshold scaling between COMPASS-D, DIII-D, and JET is presented in Ref. [17]. The linear scaling of the low density limit with error field is consistent in all machines. The weak toroidal field scaling shown in Eq. (12) agrees well with that of the JET device, but is quite different from the smaller COMPASS-D machine, where $n_{\mathrm{LM}} \propto B_{\mathrm{T}}^{1.9}$. Though not well understood, the difference is thought to be due to the strong variation of plasma rotation velocity as a function of toroidal field in the COMPASS-D tokamak.

The expressions for $B_{\text {pen }}$ shown in Eqs (13) and (14) explicitly illustrate the dependence on $q_{95}$. The value of $q_{95}$ (the surface enclosing $95 \%$ of the toroidal flux) strongly affects the spacing between neighboring rational flux surfaces and therefore the amount of mode coupling. Incorporating this effect requires two separate fits to determine the mode-coupling coefficients of Eq. (7), one for higher values, $q_{95} \geq 4.6$, and one for lower values, $q_{95} \leq 3.2$. In addition to the mode-coupling coefficients, the fits also determine Fourier coefficients of the unknown error field terms [associated with the $b_{\mathrm{mn}}$ of Eq. (10)] for low-order toroidal and poloidal mode numbers. The fit coefficients from unknown sources are used with the calculated Fourier coefficients from the known sources to determine the total error field. The relative magnitudes of the $m, n=2,1$ error field components are shown in Table 1, where discharges at high and low $q_{95}$ are compared. At higher $q$ values, the dominant error field comes from the unknown sources $\left(\vec{b}_{21} B_{\mathrm{T}}\right)$. As $q$ is lowered, the known errors from poloidal field coils $\left(\vec{B}_{210}\right)$ play a more important role. In both cases, the difference in the toroidal phases (defined clockwise looking down on the tokamak) of the two components is helpful in preventing a large total error field $\left(\vec{B}_{\mathrm{r} 21}\right)$.

Table I

\begin{tabular}{ccccccc}
\hline $\begin{array}{c}\text { Shot } \\
\text { Number }\end{array}$ & $\begin{array}{c}B_{\mathrm{T}} \\
(\mathrm{T})\end{array}$ & $\begin{array}{c}I_{p} \\
(\mathrm{MA})\end{array}$ & $q_{95}$ & $\begin{array}{c}\bar{B}_{210} \\
(\mathrm{G})\end{array}$ & $\begin{array}{c}\bar{b}_{21} B_{\mathrm{T}} \\
(\mathrm{G})\end{array}$ & $\begin{array}{c}\bar{B}_{\mathrm{T} 21} \\
(\mathrm{G})\end{array}$ \\
\hline 82927 & -2.1 & 1.6 & 4.6 & 4.2 at $334^{\circ}$ & 7.1 at $112^{\circ}$ & 4.9 at $77^{\circ}$ \\
91858 & -1.1 & 1.0 & 3.3 & 2.5 at $333^{\circ}$ & 3.6 at $112^{\circ}$ & 2.4 at $68^{\circ}$ \\
\hline \hline
\end{tabular}

The Fourier coefficients from known sources are calculated on an elliptical surface that approximates the shape of the $q=2$ surface for most plasmas. The variation in size and location of the $q=2$ surface is illustrated in Fig. 5(a), where the equilibrium fit flux surfaces for discharge 91859 , with $q_{95}=3.3$, are shown along with the superimposed $q=2$ surface from discharge 91860 , with $q_{95}=4.6$. Typical DIII-D discharges have $q_{95} \geq 4.6$, so the Fourier coefficients are calculated on the $q=2$ surface of discharge 91860. For discharges with $q_{95} \leq 3.2$, the coefficients are calculated on the $q=2$ surface of discharge 91859 . For interim values, interpolation is used to get the most accurate 
estimate for the Fourier coefficients. An example of the poloidal mode spectrum calculated from the Fourier coefficients for the error field correction coils is shown in Fig. 5(b,c), where the left-handed $n=1$ poloidal mode spectra are plotted for full current (5000 amps) in the $n=1$ coil and in a single C-coil pair. The spectrum of the $n=1$ coil is similar in amplitude, but contains higher order modes than the $\mathrm{C}$-coil. The phase of each mode component is constant for the C-coil, but varies with $\mathrm{m}$ for the $n=1$ coil. These features were exploited when the fields of the two coils are combined to allow a wide variation in the spectrum of the combined coils.

The accuracy of the regression analysis results of Eqs (12) through (14) is checked by plotting its predictions along with the data from several scans, as shown in Fig. 6. Each scan in Fig. 6 represents a subset of the low density locked mode threshold database for given values of $q_{95}$ and $B_{\mathrm{T}}$. Figure 6(a) shows scans of the $n=1$ component of the C-coil phase at near-optimum C-coil amplitude and Fig. 6(b) shows scans of the amplitude at near-optimum phase. In each case, there was no $n=1$ coil current and the scans were performed at two different toroidal fields for the high $q$ and low $q$ data sets. In Fig. 6(c), similar scans of the $n=1$ coil current are shown at three different values of the C-coil current. The curves drawn through the data are calculated using Eq. (12) and either Eq. (13) or (14), depending on the value of $q_{95}$. The shading around each curve represents the uncertainty $(\sigma)$ of the multivariate regression analysis fit of the entire database. From the scans it is evident that a minimum value for the locked mode density threshold is achieved with optimum values of the $n=1$ coil current, C-coil current, and C-coil phase.

Figure 7 shows every point in the locked mode threshold database, combined and plotted against the predictions of the model. All four sets of low density locked mode threshold data are plotted, representing a range of toroidal field, safety factor and error field harmonic content. Although the model is fairly general, the spread of data shown in Fig. 7 indicates a relatively good fit for data that spans a significant range in parameters. The model has now been incorporated into the software of the real-time plasma feedback control system.

Routine error field correction on DIII-D is accomplished by applying correction coil currents that optimize the error field $B_{\text {pen }}$ represented by Eq. (13) or (14). In most cases, a good estimate of the value of $q_{95}$ is known before a discharge is run, and the correct set of mode-coupling coefficients is pre-programmed into the error field correction algorithm. In addition, between-shot software automatically checks the programmed coefficients against the value of $q_{95}$ and alerts the operator for any needed changes so that optimum error field correction can be used on the next discharge. 

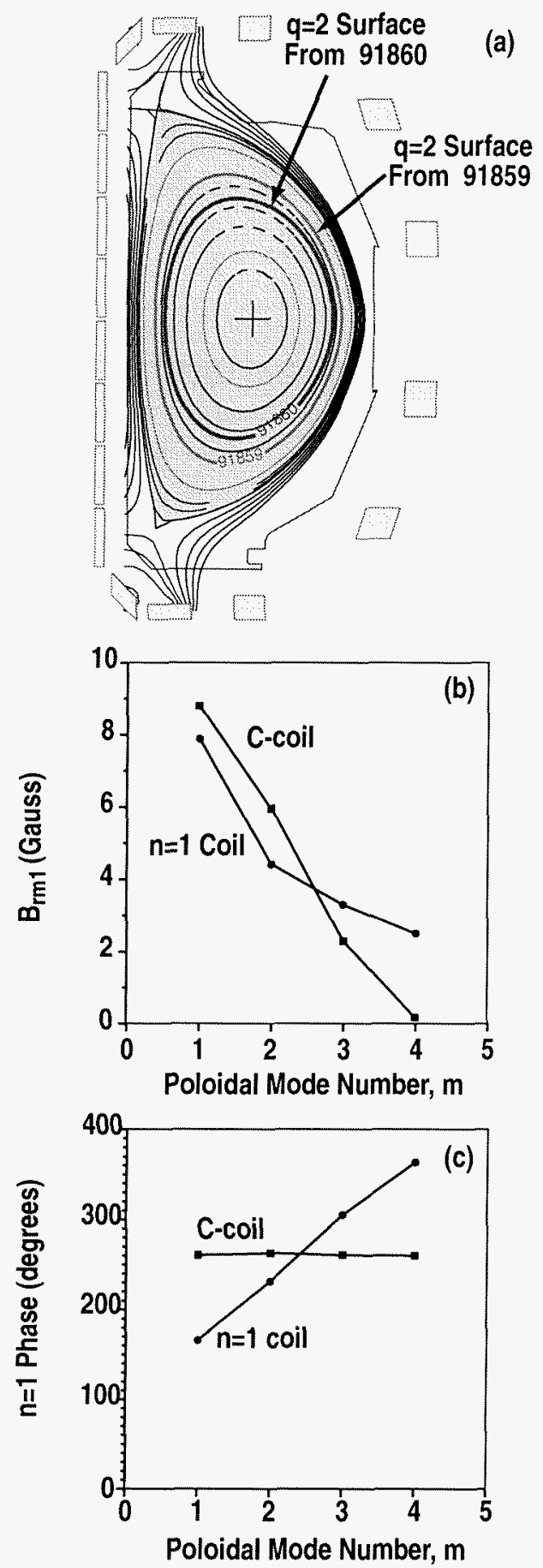

Fig. 5. (a) Flux plot showing the $q=2$ flux surface for discharge 91859 , with $q_{95}=3.3$, and discharge 91860 , with $q_{95}=4.6$. Fourier coefficients for all error field sources are calculated on one of these surfaces. (b) The left-handed $n=1$ poloidal mode spectra on the $91860 q=2$ flux surface for 5000 amperes in the $n=1$ coil and 5000 amperes in one $\mathrm{C}$-coil pair. (c) The phase spectra associated with (b).
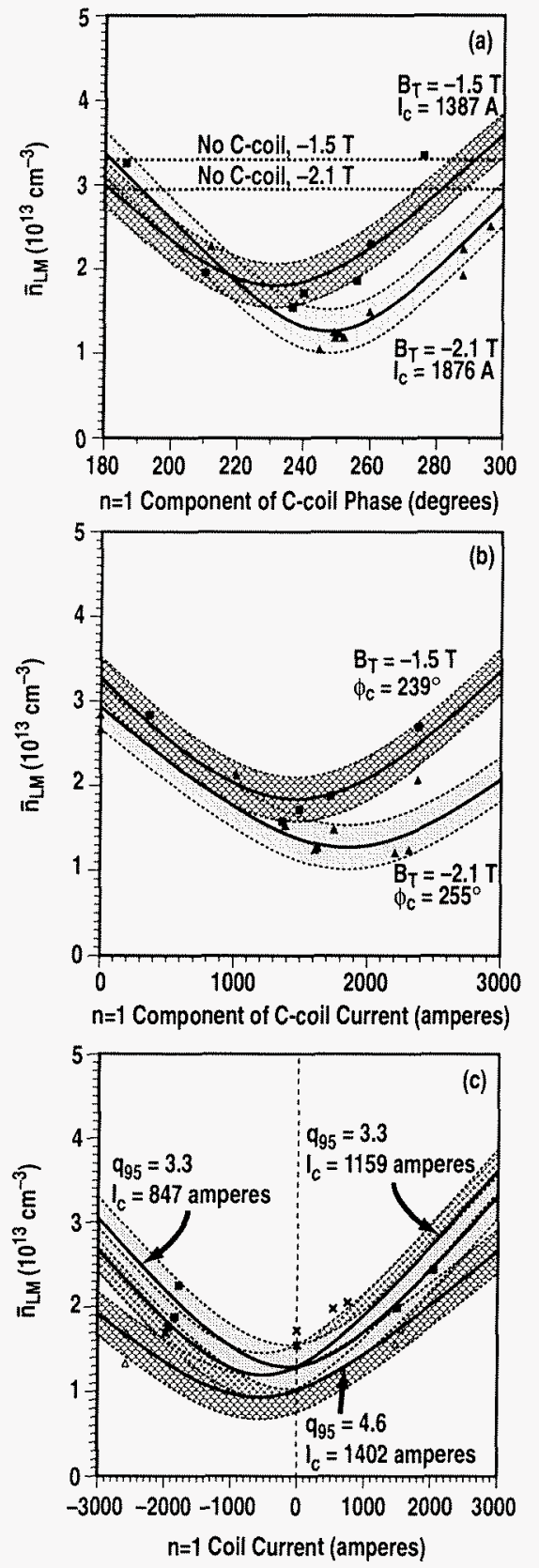

Fig. 6. In each plot, the solid curve is the regression fit of the model based on Eqs (12) through (14) for a subset of the database and dashed lines represent the $\pm \sigma$ uncertainty of the fit. (a) The locked mode threshold density vs. the phase of the C-coil $n=1$ component at near-optimum C-coil amplitude, for low and high $B_{\mathrm{T}}$. The constant $\mathrm{C}$-coil current for each data set is shown, as well as the "natural" locked mode threshold if there was no C-coil current $\left(\boldsymbol{\Lambda}: B_{\mathrm{T}}=-2.1 \mathrm{~T}, \mathbf{\square}: B_{\mathrm{T}}=-1.5 \mathrm{~T}\right)$. (b) The locked mode density vs. the $n=1$ component of the C-coil amplitude at near-optimum $C$-coil phase, for low and high $q_{95}\left(\mathbf{\Lambda}: B_{\mathrm{T}}=-2.1 \mathrm{~T}, \mathbf{\mathbf { Q }}: B_{\mathrm{T}}=-1.5 \mathrm{~T}\right)$. (c) The locked mode density vs. the $n=1$ coil current for three values of $\mathrm{C}$-coil current. $\left(\Delta: I_{\mathrm{c}}=1402\right.$ amperes, a: $I_{\mathrm{c}}=847$ amperes, $\times: I_{\mathrm{c}}=1159$ amperes). 


\section{Role of Mode-Coupling}

Incorporating the mode-coupling terms into the expression for $B_{\text {pen }}$ resulted in the need to fit a relatively large number of parameters with a regression analysis of a database of modest size. In fact, the individual errors for the mode-coupling coefficients are relatively large, as shown below:

$$
\begin{array}{lll}
q_{95} \leq 3.2 & w_{11}=0.28 \pm 0.43, & w_{31}=0.51 \pm 0.25 \\
& u_{11}=0.25 \pm 0.31, & u_{31}=-0.05 \pm 0.38 \\
q_{95} \geq 4.6 & w_{11}=0.32 \pm 0.54, & w_{31}=0.41 \pm 0.38 \\
& u_{11}=0.21 \pm 0.37, & u_{31}=0.30 \pm 0.56
\end{array}
$$

Taken individually, most of the coefficients in Eq. (15) could be zero without increasing the reduced $\chi^{2}$ of the overall fit. However, if all of the coefficients were zero, the resulting fit using only the $m, n=2,1$ mode has a reduced $\chi^{2}$ approximately 3.5 times as large as the fit that includes mode-coupling coefficients. In addition, the single-mode fit (using only the $m, n=2,1$ mode) predicts much lower density before reaching the locked mode threshold than can be realized in experiments. Clearly there was a need to incorporate additional physics to explain why the stable density limit was not as low as predicted by the single-mode fit. Including the effects of mode-coupling by using the coefficients of Eq. (15) successfully achieves this goal and accurately predicts the locked mode density limit over a wide range of plasma parameters.

\section{Recent Error Field Measurements}

Subsequent measurements made from within the vacuum vessel have tried to find the source of the anomalous $n=1$ error field inferred from the toroidal field coil [20]. Generally, it is found that the poloidal field coil components are smaller than previously reported. Preliminary analysis of the $n=1$ asymmetry from the toroidal field (TF) coil and a 
re-examination of the geometry of buswork, coil feed points, etc., does not account for the empirically deduced $n=1$ error field. Recalculation of TF coil current feeds (that produce small dipoles), connections, crossovers, etc., accounts for only approximately 1.7 Gauss of resonant left-handed $m, n=2,1$ field for the full toroidal field of 2.1 Tesla. This is about $1 / 4$ of the magnitude of the empirical measurement as seen in Table I. 


\section{CONCLUSIONS}

\section{A. Summary of Results}

The use of error field optimization on DIII-D has substantially increased the stable parameter space of the tokamak by lowering the density threshold for the onset of locked modes. Low density Ohmic locked mode experiments on the DIII-D tokamak were carried out using the combined fields of the $n=1$ coil and C-coil to produce error fields with a range of poloidal mode spectra. These experiments allowed the determination of the importance of several low order mode components in addition to the previously studied $m, n=2,1$ mode.

Results of a multivariate regression analysis of the low density locked mode threshold database produced the scaling relationship, $n_{\mathrm{LM}} \propto B_{\mathrm{T}}^{-0.01} q_{95}^{-0.79} B_{\text {pen }}$, between the locked mode density threshold and the error field. A linear dependence on the error field $B_{\text {pen }}$ is predicted, with relatively strong dependence on the safety factor and very weak dependence on the toroidal field. The fit also determined the coefficients for both viscous and direct toroidal mode coupling to the $m, n=2,1$ mode from the 1,1 and 3,1 modes. These coefficients define the form of the penetration field $B_{\text {pen }}$, as shown in Eqs (13) and (14). In addition, an unknown error field component proportional to toroidal field is calculated.

Known error fields from the poloidal field coils and the correction coils determine a set of toroidal and poloidal mode Fourier coefficients calculated on an elliptical surface that approximates the $q=2$ surface for most plasma discharges. Using these coefficients along with the empirically determined Fourier coefficients from the unknown source allows the calculation of the total error field dynamically during the plasma discharge. The value of $q_{95}$ is used to modify the Fourier coefficients to account for variation in the location of the $q=2$ surface. A set of mode-coupling coefficients from the regression analysis is chosen for either high $q_{95}$ or low $q_{95}$ discharges, with interpolation used for intermediate values of $q_{95}$. In this way, the optimum expression for the penetration field is used by the realtime plasma feedback control system, which minimizes the error field by dynamically varying the correction coil currents, leading to better plasma performance.

\section{B. Future Work}

To date, error field optimization has been determined by the ability to avoid the locked mode instability, especially at low density. Recent experiments with plasmas near the resistive wall mode (RWM) stability limit have indicated that the error field correction algorithm optimized for low density plasmas may not be ideal for rapidly rotating high $\beta$ 
plasmas [21]. A better approach for this "advanced tokamak" regime may be to determine optimum error field correction coil currents by minimizing the decay of plasma rotation. Such an approach is presently being studied, in hope that it will elucidate the complex error field picture for many types of plasma discharges. Ultimately, an error field correction algorithm should be developed that works in plasmas with high or low density, high or low $\beta$, fast or slow rotation, and along several boundaries of the stable operating parameter space of the tokamak. 


\section{REFERENCES}

[1] Karger, F., et al., in Plasma Physics and Controlled Nuclear Fusion Research 1974 (Proc. $5^{\text {th }}$ Int. Conf. Tokyo, 1974) Vol. 1, IAEA, Vienna (1975) 207.

[2] Bol, K., et al., in Plasma Physics and Controlled Nuclear Fusion Research 1974 (Proc. $5^{\text {th }}$ Int. Conf. Tokyo, 1974) Vol. 1, IAEA, Vienna (1975) 83.

[3] Hender, T.C., et al., Nucl. Fusion 32, 2091 (1992).

[4] Morris, A.W., et al., Phys. Fluids B 4, 413 (1992).

[5] Scoville, J.T., et al., Nucl. Fusion 31, 875 (1991).

[6] La Haye, R.J., et al., Phys. Fluids B 4, 2098 (1992).

[7] Fishpool, G.M., Haynes, P.S., Nucl. Fusion 34, 109 (1994).

[8] Lee, J.K., et al., Nucl. Fusion 23, 63 (1983).

[9] Fitzpatrick, R., Hender, T.C., Phys. Fluids B 3, 644 (1991).

[10] Fitzpatrick, R., Nucl. Fusion 33, 1049 (1993).

[11] Hender, T.C., et al., Nucl. Fusion 32, 2091 (1992).

[12] Jensen, T.H., Phys. Fluids B 3, 1650 (1991).

[13] Hanson, J.D., Nucl. Fusion 34, 441 (1994).

[14] La Haye, R.J., et al., Nucl Fusion 32, 2119 (1992).

[15] La Haye, R.J., Scoville, J.T., Rev. Sci. Instrum. 62, 2146 (1991).

[16] Scoville, J.T., La Haye, R.J., Bull. Am. Phys. Soc. 40 (1995).

[17] Buttery, R.J., et al., Nucl. Fusion 39, 1827 (1999).

[18] Leuer, J.A., et al., Proc. $17^{\text {th }}$ IEEE/NPSS Symposium on Fusion Engineering, San Diego, California, 1997, Vol. 1 (1998) p. 505.

[19] Buttery, R.J., et al., Nucl. Fusion 40, 807 (2000).

[20] Luxon, J.L., et al., in Proc. $22^{\text {nd }}$ Symposium on Fusion Technology, Lisbon, Portugal, 2002, to be published in Fusion Technology.

[21] Garofalo, A.M., et al., Nucl. Fusion 41, 1161 (2001). 


\section{ACKNOWLEDGMENT}

This work was supported by the U.S. Department of Energy under Contract No. DEAC03-99ER54463. 